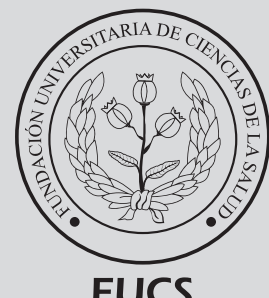

\title{
Predicción del COVID-19 a nivel mundial para el año 2021
}

\section{COVID-19 worldwide forecasts for the year 2021}

${ }^{a}$ Ingeniero. Magister en Gestión de la Tecnología Educativa, Especialista en Administración de la Informática Educativa. Docente de matemáticas e Investigador, Secretaría de Educación de Soacha, Cundinamarca.

\section{R E S U M EN}

Introducción: el síndrome respiratorio agudo severo coronavirus 2 (SARS-CoV-2), el virus que origina la enfermedad por coronavirus 2019 (COVID-19), se ha difundido con rapidez por todo el mundo desde que surgió en Wuhan, China a fines de 2019. Objetivo: presentar una predicción del contagio de personas y personas fallecidas por el COVID-19 a nivel mundial para el año 2021. Metodología: el método utilizado para calcular el pronóstico fue el modelo de HOLT, procesando la información con el paquete estadístico SPSS, versión 25.0. Resultados: de acuerdo con la proyección del COVID-19 para el año 2021 a nivel mundial, se estimó que al finalizar el mes de enero de 2021 se proyecta un número de 96'878.746 personas contagiadas por COVID-19 hasta llegar a finalizar el mes de diciembre con 283'662.031 contagios. Conclusión: el modelo de HOLT nos ilustra un escenario posible de la enfermedad en cuanto a personas contagiadas y fallecidas a nivel mundial para el año 2021, lo cual a la luz de los resultados no es nada halagador para el mundo, por lo tanto se hace necesario continuar con las medidas de aislamiento para que se genere una estabilización de la enfermedad.

Palabras clave: COVID-19, predicción, SARS-CoV-2, pandemia.

(C) 2020 Fundación Universitaria de Ciencias de la Salud - FUCS. Este es un artículo Open Access bajo la licencia CC BY-NC-ND (http://creativecommons.org/licenses/by-nc-nd/4.0/).

Historia del artículo:

Fecha recibido: noviembre 17 de 2020 Fecha aceptado: diciembre 18 de 2020

\section{INFORMACIÓN DEL ARTÍCULO}

\footnotetext{
Autor para correspondencia.

Jorge Enrique Díaz Pinzón jediazp@unal.edu.co
}

DOI

10.31260/RepertMedCir.01217372.1143 
Introduction: Severe acute respiratory syndrome coronavirus 2 (SARS-CoV-2), the virus that causes coronavirus disease 2019 (COVID-19), has spread rapidly around the world since it emerged in Wuhan, China in late 2019. Objective: to describe COVID-19 worldwide forecasts of infections and deaths over the year 2021. Methodology: the method used to calculate the prediction was the Holt model. Data was analyzed with the SPSS version 25.0 statistical package. Results: according to the COVID-19 global projection for the year 2021, there will be an estimated 96' 878.746 cases by the end of January 2021 and $283^{\prime} 662.031$ by the end of December 2021. Conclusion: the HOLT model illustrates a possible scenario of the disease in terms of people infected and killed in year 2021, which in light of the results is not at all flattering for the world, so isolation measures need to continue in order to achieve stabilization of the disease.

Key words: COVID-19, prediction, SARS-CoV-2, pandemic

(C) 2020 Fundación Universitaria de Ciencias de la Salud - FUCS. This is an open access article under the CC BY-NC-ND license (http://creativecommons.org/licenses/by-nc-nd/4.0/).

INTRODUCCIÓN

El síndrome respiratorio agudo severo coronavirus (SARSCoV-2), el virus que origina la enfermedad por coronavirus 2019 (COVID-19), se ha dilatado rápidamente por todo el mundo desde que surgió en Wuhan, China a fines de 2019. ${ }^{1}$ La comprensión actual de COVID-19 procede en gran parte de la vigilancia de enfermedades y los estudios epidemiológicos elaborados en las primeras fases de la pandemia en China ${ }^{1-3}$ y los países de altos ingresos de Europa ${ }^{4,5}$ y América del Norte. ${ }^{6-8}$ La Organización Mundial de la Salud ha señalado la rápida propagación de COVID-19 en todo el mundo como una emergencia de salud pública mundial. Es bien sabido que la propagación de la enfermedad está influenciada por la gente, la voluntad de adoptar conductas preventivas de salud pública que a menudo se asocian con la percepción pública del riesgo. ${ }^{9} \mathrm{De}$ acuerdo con Chung y col. citado por Díaz ${ }^{10}$, no concurre una solución única para todos. Sin embargo, algunos ejercicios frecuentes de distanciamiento social y encierro ciertamente alcanzan aplanar la curva del aumento de los casos confirmados y las tasas de mortalidad, son las especificidades locales de muchos otros tipos, como las organizaciones económicas, los paradigmas de gobernanza y las distinciones culturales, las que establecen el éxito de la enfermedad lidiando contra los esfuerzos.

El objetivo de este trabajo de investigación es presentar una perspectiva del contagio de personas recuperadas y fallecidas por el COVID-19 en Colombia para 2021; el método utilizado para calcular el pronóstico fue el modelo ARIMA $(0,1,0)$, teniendo en cuenta el registro de la información por parte del Instituto Nacional de Salud ${ }^{10}$, hasta el 6 de noviembre 2020 punto de vista económico, social y de salud.

\section{METODOLOGÍA}

Según Hurtado y Toro (1998) citado por Díaz ${ }^{11}$, el trabajo de investigación se realizó mediante un enfoque cuantitativo; la investigación cuantitativa es aquella en la que se almacenan y exploran datos cuantitativos sobre variables y estudia las propiedades y fenómenos cuantitativos.

\section{MODELO DE HOLT}

Este modelo añade al de suavizamiento exponencial un mecanismo por la tendencia que logre tener la variable pronosticada, razón por la cual suele entenderse como suavizamiento exponencial doble. ${ }^{12-14}$

Contiene además de la constante de suavizamiento $\alpha$, una de tendencia, denominada $\delta$ o $\beta$, que en este texto usaremos $\beta$. La variable pronosticada para el periodo de tiempo $t$, se deduce con la ecuación siguiente ${ }^{12}:\left(\mathrm{Y}_{\mathrm{t}}\right)=\mathrm{L}_{\mathrm{t}}+\mathrm{pT}_{\mathrm{t}}(1),\left(\mathrm{Y}_{\mathrm{t}}\right.$ )$=\mathrm{L}_{\mathrm{t}}+\mathrm{pT}^{\mathrm{t}}(1)$, donde $\mathrm{d}\left(\mathrm{Y}_{\mathrm{t}}\right)=$ Valor pronosticado para el periodo $\mathrm{t} \mathrm{L}=$ Valor estimado para el periodo $\mathrm{t} \mathrm{T}_{\mathrm{t}}=$ Valor de la tendencia en el periodo $\mathrm{t} p=$ Periodos a pronosticar en el futuro.

Ahora, el valor estimado de la variable en el periodo $\mathrm{t}$ se consigue con la ecuación: $\mathrm{L}_{\mathrm{t}}=\mathrm{aY}_{\mathrm{t}-1}+(1-\mathrm{a})\left(\mathrm{L}_{\mathrm{t}-1}+\mathrm{Tt}-1\right)(2)$, siendo a la constante de suavizado, cuyo valor se ubica entre cero y la unidad y $\mathrm{Y}_{\mathrm{t}-1}$ el valor de la variable en el periodo t-1.

La tendencia $T_{t}$ en cualquier periodo se logra: $T_{-}$ ${ }_{t}=\beta\left(L_{t}-L_{t-1}\right)+(1-\beta) T_{t-1}(3)$, siendo $\beta$ la segunda constante de suavizamiento por modificación de la tendencia, cuyo valor también se sitúa entre cero y la unidad.

De hecho, para las dos constantes se propone manejar valores entre 0.05 y 0.50 .

Los valores idóneos serán aquellos que mengüen la sumatoria del cuadrado de los errores, siendo cada error la diferencia entre el valor real de la variable pronosticada y su valor pronosticado. ${ }^{12}$ 
Esta investigación se centró en la población de contagiados, y personas fallecidas por COVID-19 a nivel mundial con la información proveniente del tablero COVID-19 por el Centro de Ciencia e Ingeniería de Sistemas (CSSE) de la Universidad
Johns Hopkins $(\mathrm{JHU})^{15}$, en el periodo acumulado hasta el 16 de noviembre de 2020. Se utilizó el paquete estadístico SPSS v.25, para realizar el pronóstico (figuras 1 y 2).

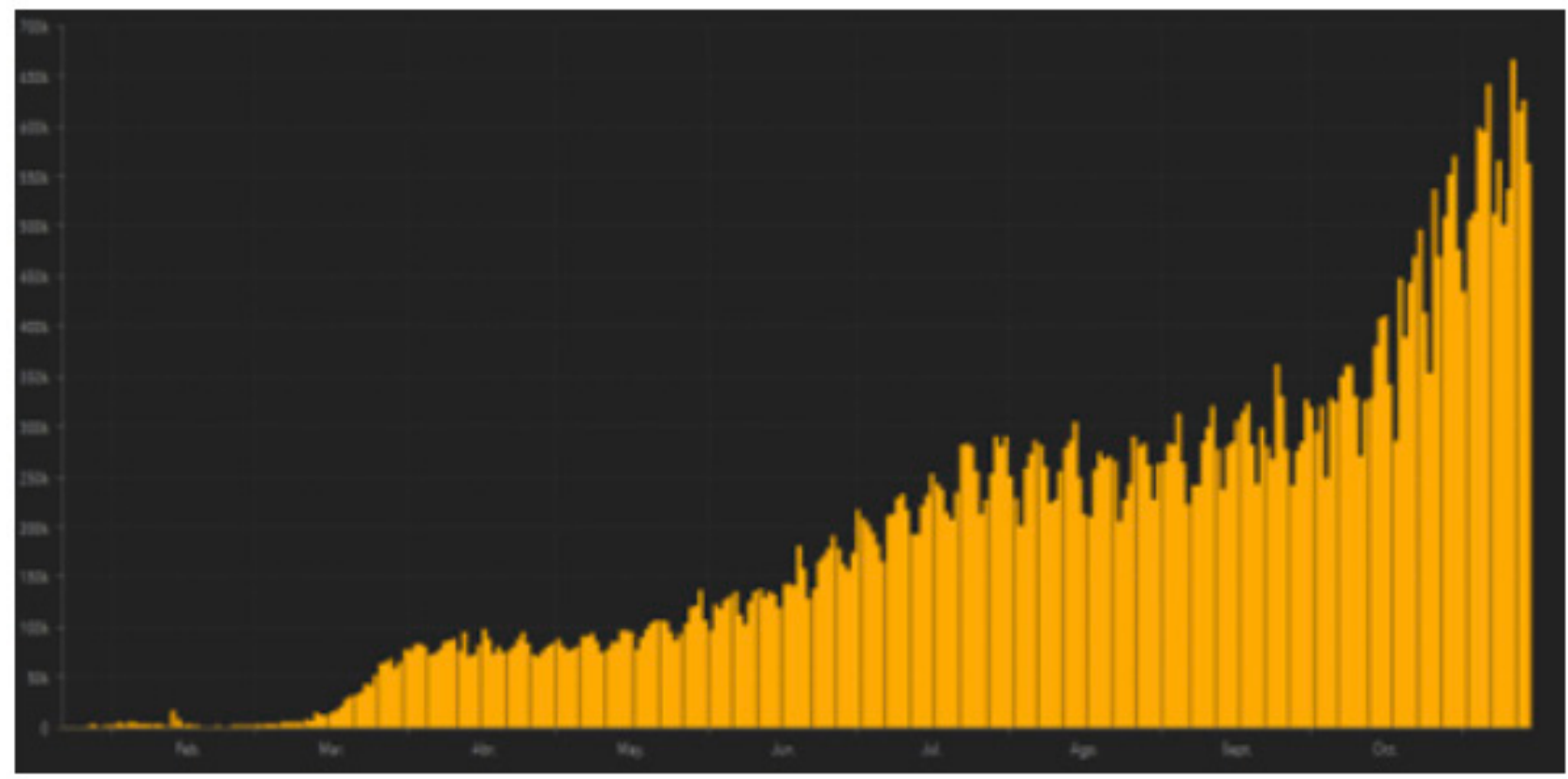

Fuente: Universidad Johns Hopkins

Figura 1. Casos diários por COVID-19 a nível mundial.

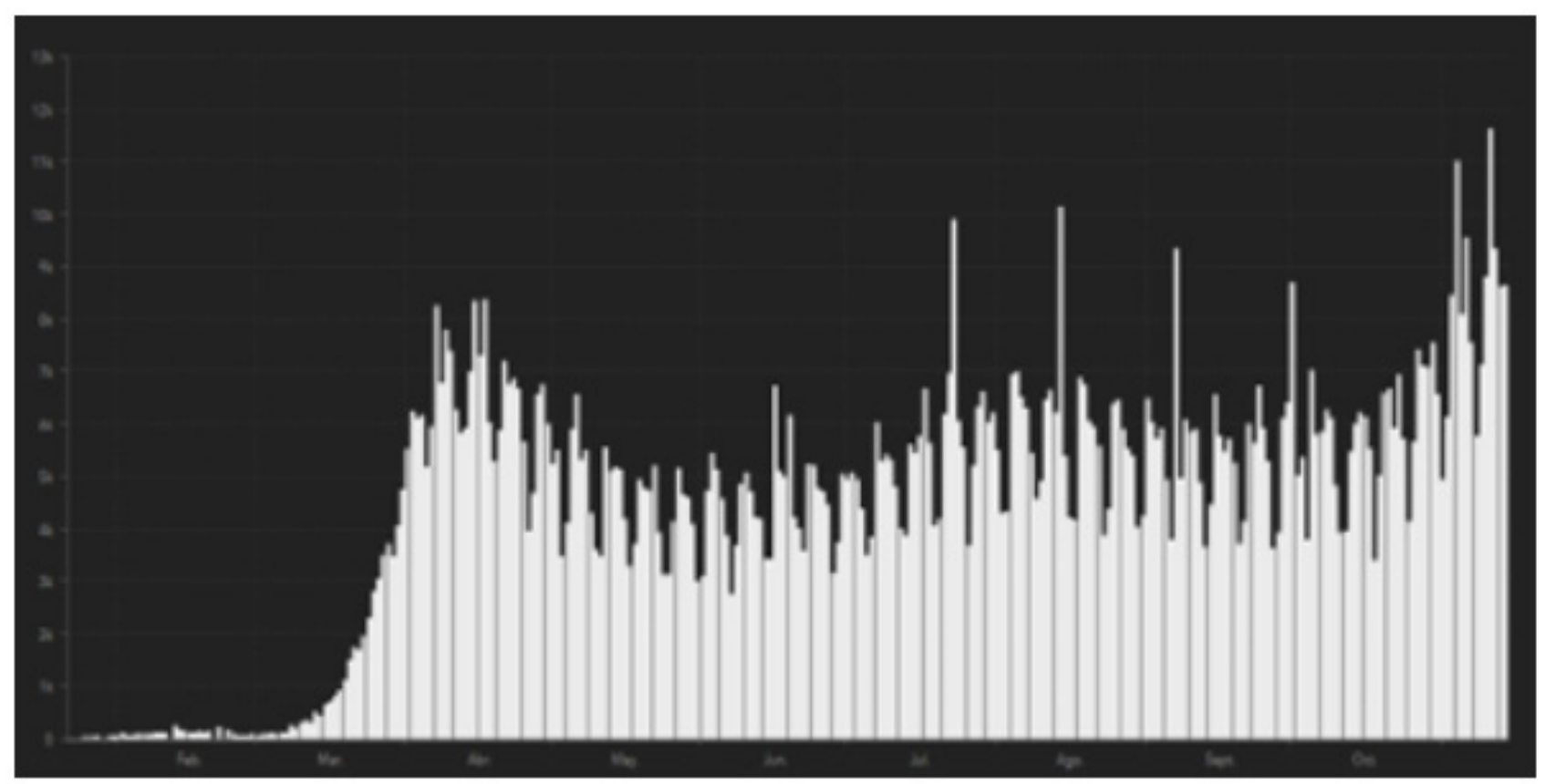

Fuente: Universidad Johns Hopkins

Figura 2. Fallecidos diários por COVID-19 a nível mundial. 
En la figura 2 apreciamos la tendencia de personas fallecidas a nivel mundial por día, comenzando desde el 2501-2020, con 14 personas fallecidas, alcanzando su pico más alto el 11-11-2020 con 11.624 personas fallecidas día y el 1611-2020 se presentó una población de fallecidos de 425.920 . El dato acumulado de fallecidos a esta fecha es de 1'318.613

\section{RESULTADOS}

En la tabla 1 se observa la descripción del modelo de Holt para los datos acumulados para personas contagiadas y fallecidas por COVID-19 a nivel mundial. El R2 coeficiente de determinación nos indica en qué medida los datos se ajustan al modelo seleccionado, modelo de HOLT, en este caso el R2 $=0,996$, y si se convierto a porcentaje se va obtener 99.6\%; esto significa que los datos se ajustan en $99,6 \%$ al modelo seleccionado para la proyección de personas contagiadas a nivel mundial para el año 2021, y un $\mathrm{R}^{2}=0,995$, esto significa que los datos se ajustan en $99,5 \%$ para la proyección de personas fallecidas a nivel mundial.

Se aprecia que el error de pronóstico fue muy bajo $\mathrm{y}$ correspondió al MAPE (error porcentual medio absoluto) con un $0,27 \%$ para la predicción de personas contagiadas, ahora, para el caso de personas fallecidas con un $0,17 \%$ es decir que en ambos casos la predicción tiene un alto grado de confiabilidad.

Tabla 1. Descripción del modelo ARIMA

\begin{tabular}{|l|l|llll|}
\hline & & & & R2 & MAPE \\
\hline ID de modelo & Contagiados-Acu & Modelo_1 & HOLT & 0,996 & 0,272 \\
ID de modelo & Recuperados-Acu & Modelo_1 & HOLT & 0,995 & 0,173 \\
\hline
\end{tabular}

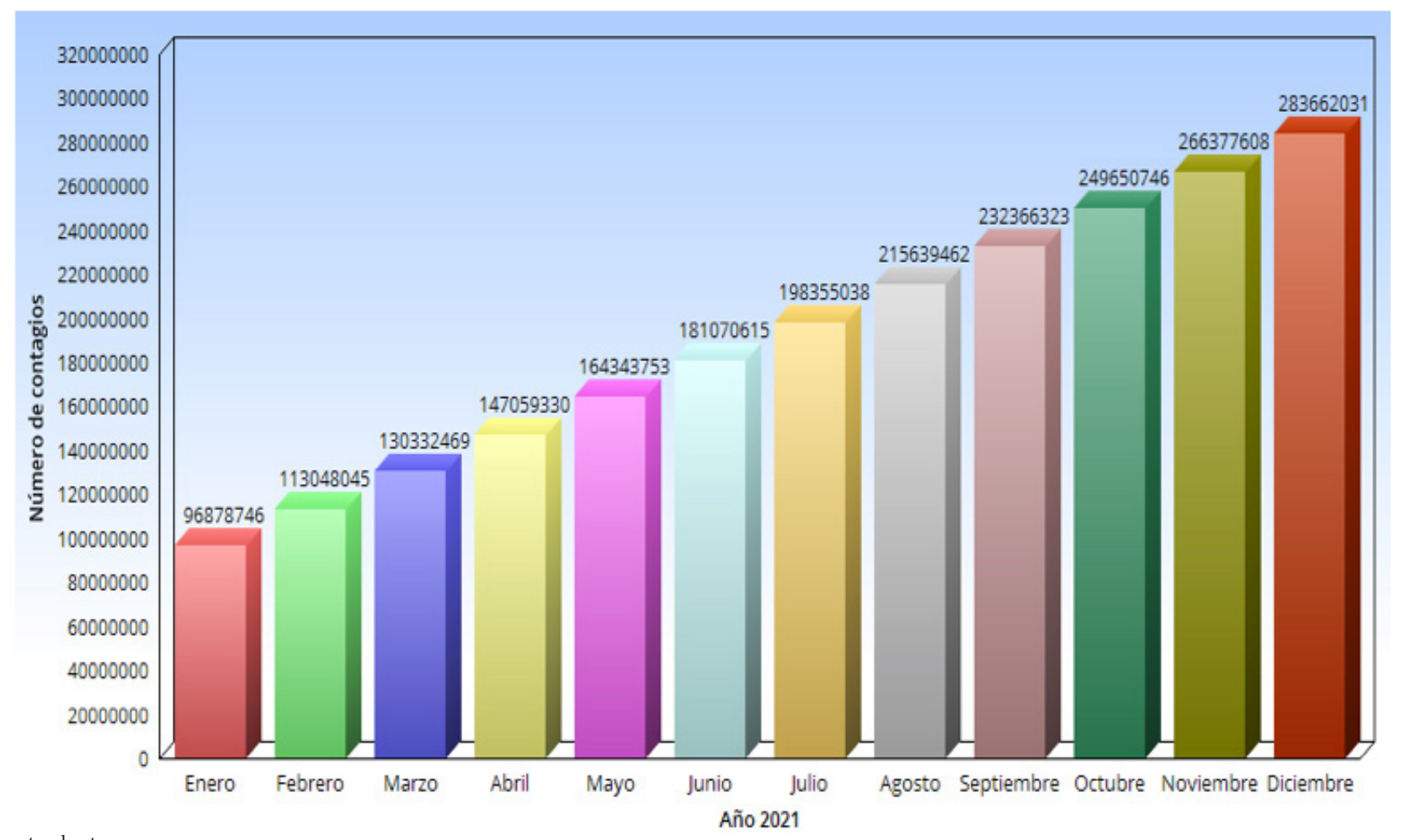

Fuente: el autor

Figura 3 Número de contagiados a nivel mundial por COVID-19 para el año 2021.

En la figura 3 se aprecia la proyección de personas contagiadas a nivel mundial para el año 2021. Al finalizar el mes de enero 2021 se proyecta un número de 96'878.746 contagiadas por COVID-19 hasta llegar a finalizar el mes de diciembre con 283'662.031.

En la figura 4 se evidencia la proyección de personas fallecidas a nivel mundial para el año 2021. Al finalizar el mes de enero de 2021 se proyecta un número de 1'957.088 fallecidas por COVID-19 hasta llegar a finalizar el mes de diciembre con 4'759.045.
En la figura 5 se observa la proyección de los 10 países con mayor número de personas contagiadas a nivel mundial para el año 2021.

Encabeza la lista Estados Unidos que al finalizar el año 2021, tendrá una población de contagiados de 57'470.801, India con 46'520.017, Brasil con 30'526.103, Rusia con 10'626.630, Francia con 9'974130, España con 7'594.131, Reino Unido con 7'147.899, Argentina con 6'823.051, Colombia con 6'241.252, e Italia con 6'135.993. 


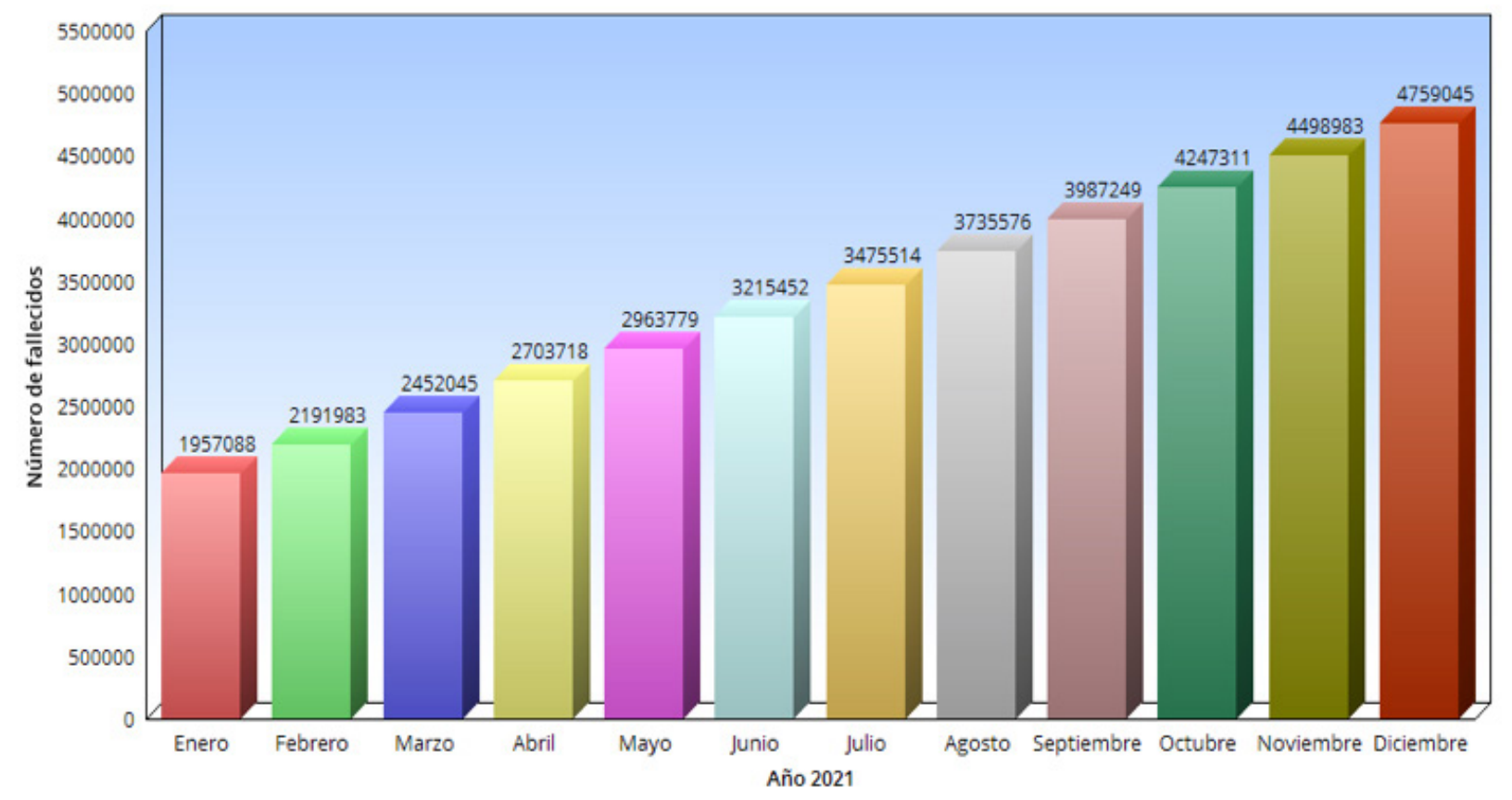

Fuente: el autor

Figura 4 Número de fallecidos a nivel mundial por COVID-19 para el año 2021

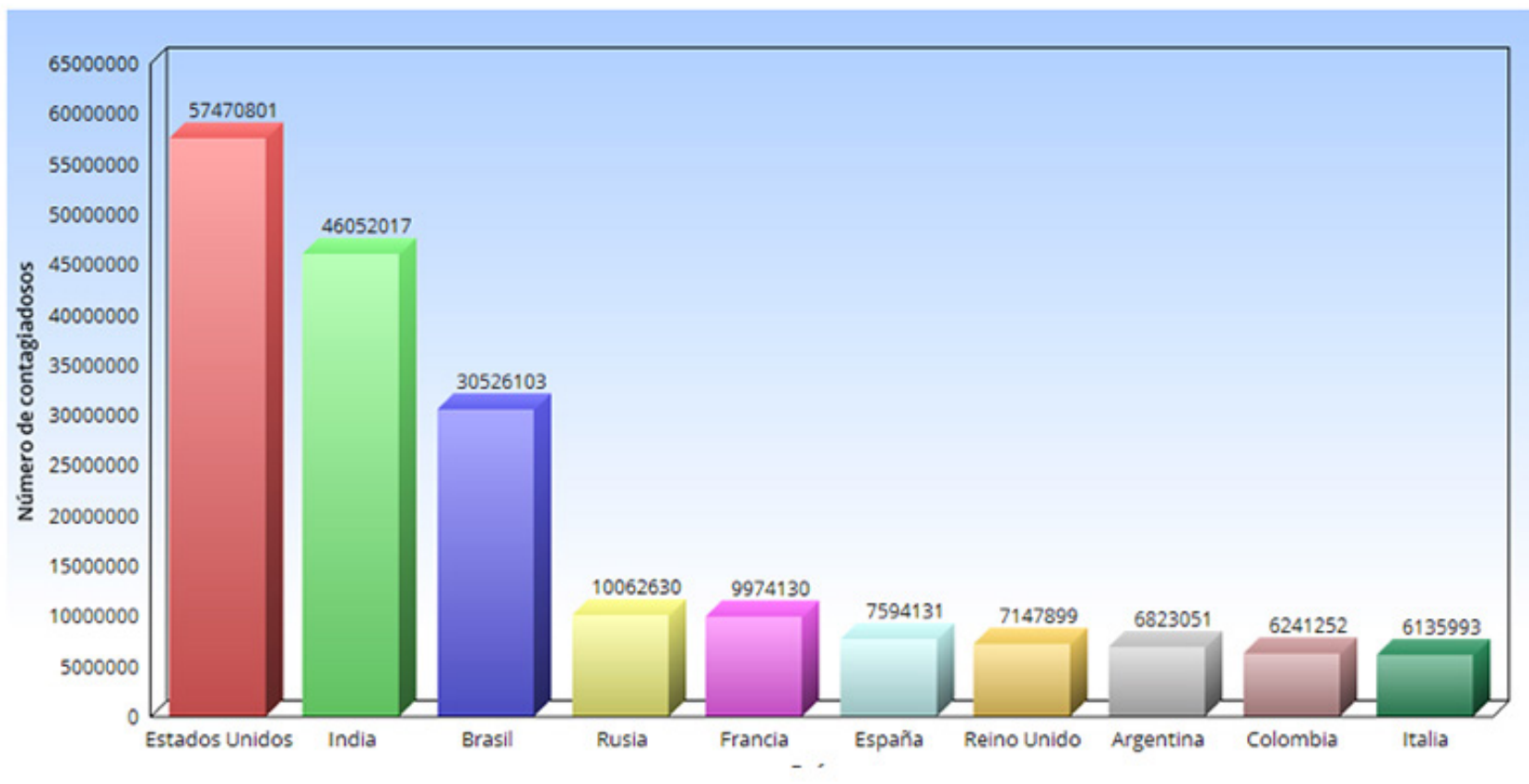

Fuente: el autor

Figura 5 Número de contagiados por países por COVID-19 para el año 2021.

En la figura 6 se observa la proyección de los 10 países con mayor número de personas fallecidas a nivel mundial para el año 2021. Encabeza la lista Estados Unidos que al finalizar el año 2021 tendrá una población de fallecidos de 888.654, Brasil con 598.386, India con 469.439, Reino Unido con 187.768, Italia con 163.237, Francia con 153.752, España con 147.140, Argentina con 127.893, Colombia con 122.822, y Rusia con 119.765. 


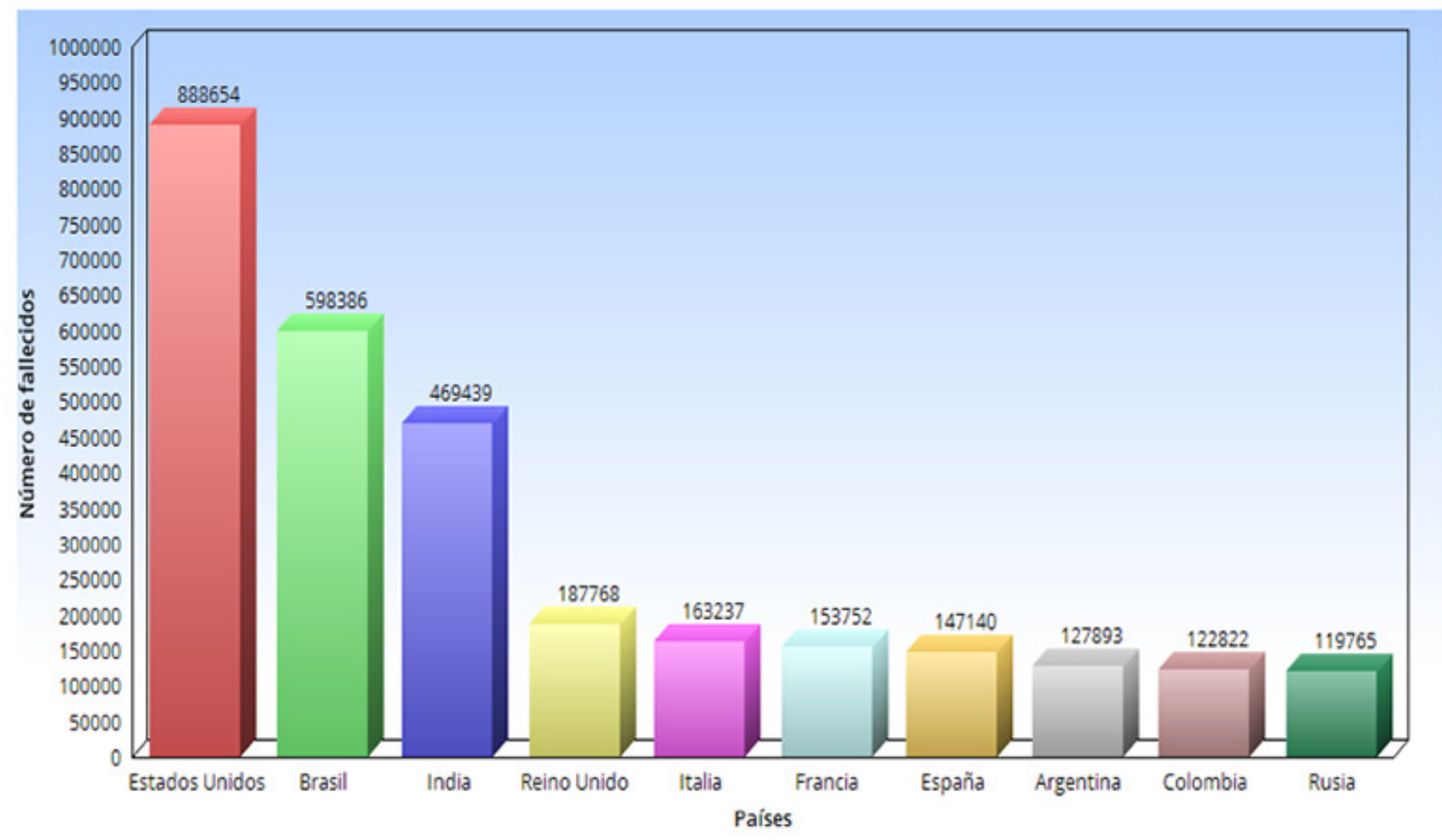

Fuente: el autor

Figura 6 Número de personas fallecidas por países por COVID-19 para el año 2021.

\section{CONCLUSIONES}

El modelo de HOLT nos ilustra un escenario posible de la enfermedad en cuanto a personas contagiadas y fallecidas a nivel mundial para el año 2021, lo cual a la luz de los resultados no es nada halagador para el mundo, por lo tanto se hace necesario continuar con las medidas de aislamiento para que se genere una estabilización de la enfermedad. Esta predicción puede cambiar si todos los países a nivel mundial son responsables seguir con las medidas de prevención de contagio para el SARS-CoV-2.

Además, según Coutin citado por Díaz ${ }^{16}$ la utilización de modelos de predicción de fácil construcción comparativa como los obtenidos con alisamientos exponenciales en este estudio, logran resultar muy útiles para la vigilancia de eventos de salud diversos ya que admiten a las autoridades sanitarias el conocimiento previo que facilita en gran medida la toma de decisiones oportunas.Se apreció que el error de pronóstico fue muy bajo, y correspondió al MAPE (error porcentual medio absoluto) con un 0,27\%, para la predicción de personas contagiadas. Para el caso de personas fallecidas con un $0,17 \%$ es decir que en ambos casos la predicción tiene un alto grado de confiabilidad.

De acuerdo con la proyección del COVID-19 para el año 2021 a nivel mundial por el modelo de HOLT, se estimó que al finalizar el mes de enero de 2021 se proyecta un número de
96'878.746 personas contagiadas por COVID-19 hasta llegar a finalizar el mes de diciembre con 283'662.031 contagios. También se evidenció la proyección de personas fallecidas a nivel mundial para el año 2021. Al finalizar el mes de enero de 2021 se proyecta un número de 1'957.088 personas fallecidas por COVID-19 hasta llegar a finalizar el mes de diciembre con 4'759.045 fallecimientos. La utilización de modelación matemática ha prosperado en grado representativo en las últimas décadas y es de gran empuje para ilustrar escenarios capaces de prevención y control de enfermedades infectocontagiosas, con la finalidad de seguir haciendo un monitoreo del SARS-CoV-2 y poder contrarrestar su velocidad de propagación a nivel mundial. ${ }^{17}$

\section{REFERENCIAS}

1. Li Q, Guan X, Wu P, Wang X, Zhou L, Tong Y, et al. Early Transmission Dynamics in Wuhan, China, of Novel Coronavirus-Infected Pneumonia. N. Engl. J. Med.. 2020;382(13):1199-207. doi: 10.1056 / NEJMoa2001316

2. Zhou F, Yu T, Du R, Fan G, Liu Y, Liu Z, et al. Clinical course and risk factors for mortality of adult inpatients with COVID-19 in Wuhan, China: a retrospective cohort study. Lancet. 2020;395(10229):1054-62. doi: 10.1016/S0140-6736(20)30566-3 
3. Hoffmann M, Kleine-Weber H, Schroeder S, Kruger N, Herrler T, Erichsen S, et al. SARS-CoV-2 Cell Entry Depends on ACE2 and TMPRSS2 and Is Blocked by a Clinically Proven Protease Inhibitor. Cell. 2020;181(2):27180 e8. doi: https://doi.org/10.1016/j.cell.2020.02.052

4. Grasselli G, Zangrillo A, Zanella A, Antonelli M, Cabrini L, Castelli A, et al. Baseline Characteristics and Outcomes of 1591 Patients Infected With SARS-CoV-2 Admitted to ICUs of the Lombardy Region, Italy. Jama. 2020;323(16):1574-81. doi: 10.1001 / jama.2020.5394

5. Docherty AB, Harrison EM, Green CA, Hardwick HE, Pius R, Norman L, et al. Features of 20133 UK patients in hospital with covid-19 using the ISARIC WHO Clinical Characterisation Protocol: prospective observational cohort study. BMJ. 2020;369:m1985. doi: 10.1136/bmj. m1985

6. Richardson S, Hirsch JS, Narasimhan M, Crawford JM, McGinn T, Davidson KW, et al. Presenting Characteristics, Comorbidities, and Outcomes Among 5700 Patients Hospitalized With COVID-19 in the New York City Area. Jama. 2020;323(20):2052-9. Epub 2020/04/23.

7. Petrilli CM, Jones SA, Yang J, Rajagopalan H, O'Donnell L, Chernyak Y, et al. Factors associated with hospital admission and critical illness among 5279 people with coronavirus disease 2019 in New York City: prospective cohort study. BMJ. 2020;369:ml966.

8. Lewnard JA, Liu VX, Jackson ML, Schmidt MA, Jewell BL, Flores JP, et al. Incidence, clinical outcomes, and transmission dynamics of severe coronavirus disease 2019 in California and Washington: prospective cohort study. BMJ. 2020;369:m1923.

9. Dryhurst S, Schneider CR, Kerr J, Freeman ALJ, Recchia G, van der Bles AM, et al. Risk perceptions of COVID-19 around the world. Journal of Risk Research. 2020;23(7-8):994-1006.
10. Díaz-Pinzón, J.E. (2020). Estudio de los resultados del contagio por COVID-19 a nivel mundial. Repert Med Cir. 2020;29(Supl. Núm. 1):65-71. DOI: 10.31260/RepertMedCir.01217372.1089

11. Díaz-Pinzón, J.E. Correlación y regresión lineal de la evaluación tiempo y puntaje con recurso interactivo flash. INNOVA Research Journal. 2017;2(10):1-8. doi: https://doi.org/10.33890/innova.v2.n10.2017

12. Betancourt D. La tendencia en el suavizamiento exponencial doble o modelo de Holt [Internet]. Ingenio Empresa; 2020 [cited 2020 noviembre 13]; Available from: https://ingenioempresa.com/ suavizacion-exponencial-doble/.

13. Izar JM. Método de Holt [Internet]. ResearchGate; 2019 [cited 2020 noviembre 3]; Available from: https://www.researchgate.net/ publication/331844153_Metodo_de_Holt.

14. Camacho A. Método Holt [Internet]. slideshare; 2011 [cited 2020 noviembre 16]; Available from: https://es.slideshare.net/adzarael/ mtodo-holt.

15. Universidad Johns Hopkins. COVID-19 Dashboard by the Center for Systems Science and Engineering (CSSE) at Johns Hopkins University (JHU) [Internet]. Universidad Johns Hopkins; 2020 [cited 2020 noviembre 16]; Available from: https://www.arcgis.com/apps/opsdashboard/index.html\#/ bda7594740fd40299423467b48e9ecf6.

16. Díaz-Pinzón JE. Uso de modelo predictivo para la dinámica de transmisión del COVID-19 en Colombia. Repert Med Cir. 2020;29(Núm. Supl.1):34-44. doi: 10.31260/RepertMedCir.01217372.1056

17. Díaz-Pinzón JE. Proyección de la propagación del COVID-19 en Colombia. Rev. Med. 2020;28(1):11-20. doi: 10.18359/ rmed.4702 